\title{
CODEE Journal
}

Volume 8

Article 4

$3-31-2011$

\section{ODE Architect: Now and Future}

Jeho Park

Follow this and additional works at: http://scholarship.claremont.edu/codee

\section{Recommended Citation}

Park, Jeho (2011) "ODE Architect: Now and Future," CODEE Journal: Vol. 8, Article 4.

Available at: http://scholarship.claremont.edu/codee/vol8/iss1/4

This Article is brought to you for free and open access by the Journals at Claremont at Scholarship @ Claremont. It has been accepted for inclusion in CODEE Journal by an authorized administrator of Scholarship@ Claremont. For more information, please contact scholarship@cuc.claremont.edu. 


\title{
ODE Architect: Now and Future
}

Jeho Park

Harvey Mudd College

Keywords: ODE Architect, Virtualization, Cloud computing

Manuscript received on January 17, 2011; published on March 31, 2011.

\begin{abstract}
This article describes current compatibility issues with ODE Architect software suite and 64-bit Windows operating systems, and proposes viable alternatives using virtual machine environment and cloud computing.
\end{abstract}

\section{Introduction}

Computer software and hardware evolve rapidly; some are extinct early and some are prolonged longer than others by adapting themselves into the fast-paced evolution of computing environment. In between those two groups, the ceased or the evolved, there is another group of software called legacy software. For some computer applications, users want to keep using the software regardless of its oldness because the software is still useful for their computing needs. But such legacy software users are prone to having incompatibility issues whenever they purchase new computers or install new operating systems.

ODE Architect (ODEA) is a legacy software package which first appeared in 1998 under support of the Consortium of ODE Experiments ${ }^{1}$ (CODEE) and the National Science Foundation. Although it is a 12-year-old program, it is known to provide useful teaching environment for college-level Differential Equations courses. The ODEA package is bundled with books, Differential Equations: A Modeling Perspective [2], written by Harvey Mudd College Professors Robert Borrelli and Courtney Coleman, and Elementary Differential Equations [1] written by William Boyce and Richard DiPrima. It is also sold as an independent software package through John Wiley and Sons, Inc. ${ }^{2}$ The book written by two Math Professors at Harvey Mudd College is being actively used in several differential equations classes at HMC and Pomona College as of this writing. So the incompatibility issues of the ODEA package are a current problem for HMC students and faculty who wish to try the software for their teaching and learning along with the text.

In this article, we describe the current issues with ODEA package for running in modern operating systems and propose viable alternatives, virtualization and cloud computing, to use the legacy educational software package now and in the future.

\footnotetext{
1http://www.codee.org/

${ }^{2}$ http: //www.wiley.com/WileyCDA/WileyTitle/productCd-047143955X.html
} 


\section{Background: ODE Architect Software Package}

From 1992-1997, the Consortium of ODE Experiments (CODEE), was supported by the National Science Foundation with the goal of sharing the rapidly growing wealth of computational instruction techniques with as many teachers and students of differential equations as possible. To accomplish that goal CODEE published a newsletter that provided a regular source of ideas, inspiration, and experiments for instructors of ODEs. Past issues of the CODEE Newsletters are available at the CODEE Digital Library. ${ }^{3}$

Because computers are vital tools in helping students understand and visualize concepts in differential equations, CODEE also produced a software solver package called ODE Architect. which appeared in 1998 and is currently marketed by John Wiley \& Sons. ODE Architect (ODEA) is a Windows software package that provides a highly interactive software environment for constructing and exploring the user's own ODE models of realworld phenomena. ODE Architect has three components: the ODE Architect Tool, the Multimedia ODE Architect, and the ODE Architect Library. ODE Architect was designed to be easy to use. Work on the CODEE project ${ }^{4}$ was headquartered at Harvey Mudd College and involved faculty from 7 institutions in the U.S.A.

The ODEA Tool employs a graphical user interface to enter and edit equations, control solver settings and features, and to create and edit a wide variety of $2 \mathrm{D}$ and $3 \mathrm{D}$ graphics. Figure 1 shows a screen capture of the ODEA Tool. Navigational paths are clearly marked and simple to follow using a point-and-click technique. At any place in the package the user can call up content menus and access material in any order. A second mode of operation, the Expert Mode, provides access to more advanced features.

The Tool solves systems of up to ten first-order ODEs, which are entered using a simple, natural scripting language. A solver/grapher feature for discrete dynamical systems is also available. A variety of engineering functions such as square waves, sawtooth waves and step functions are included in the Tool function library. Auxiliary functions involving the state variables can be defined and used. Graph scales can be set automatically or manually. Numerical values of solutions can be viewed in tabular form. Graphs are editable and the user can scale and label axes, mark equidistant-in-time orbital points, color the graphs, change the line styles, overlay graphs of functions and solution curves for different ODEs-all with no programming or special commands to remember.

Multimedia ODE Architect illustrates the modeling process in detail, with dozens of examples, each with its own theme, supported by highly interactive simulations. Students explore the modeling process via "what-if" scenarios and exercises. Along the way, students are guided to build their own ODEs which model a given situation and then solve them numerically and graphically. The ODEA Library has dozens of pre-programmed, editable, and interactive ODE files covering a wide range of topics from mathematics, physics, chemistry, population biology, and epidemiology. There are also many ODEs to illustrate points such as data compression, ODEs with singular coefficients, bifurcation, limit cycles, and so on. Each Library file has explanatory text along with the ODEs and includes illustrative graphs. The Library files are organized into folders with descriptive

\footnotetext{
${ }^{3}$ http: / / www. codee.org

${ }^{4}$ http: //www.math.hmc.edu/resources/odes/codee
} 


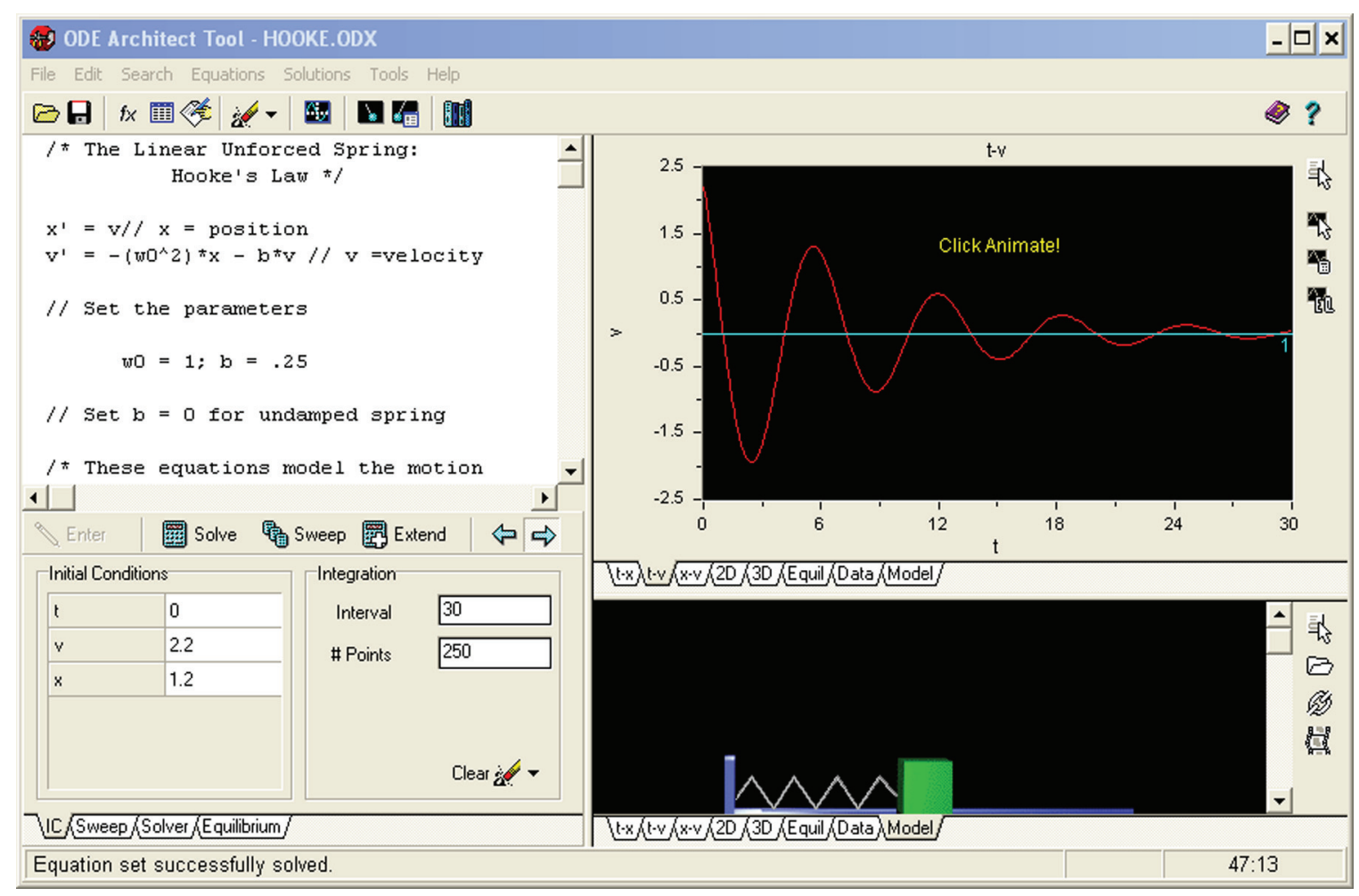

Figure 1: ODE Architect Tool's Graphical User Interface

titles to facilitate browsing. These files also provide a marvelous way to learn how to use the Architect Tool.

\section{Current State of ODE Architect: Incompatibility Is- sues}

In the summer of 2010, the former PI of the CODEE project, Professor Robert Borrelli, reported that he had received numerous reports that ODE Architect did not work under the standard release of Windows 7. To resolve the incompatibility issue of ODE Architect package in Windows 7 , he applied for the Summer Teaching with Technology Innovation Grant offered by Computing and Information Services (CIS) at Harvey Mudd College. His proposal was approved to receive technical support from CIS.

With the program's support, exhaustive testing and research had been conducted to evaluate ODE Architect on various Windows versions to find the reason for not functioning on Windows 7. As a result, we have found a couple of facts to explain the reason of this incompatibility:

1. ODE Architect including its installation program is a 16-bit application.

Due to the fact that ODE Architect installer and GUI are 16-bit applications, ODE Architect does not run on any 64-bit Windows regardless of the Windows versions (e.g. XP, Vista, 
or Windows 7) because 64-bit Windows doesn't have a 16-bit emulator. Hence, the issue statement should be altered into "ODE Architect's incompatibility with 64-bit Windows" from its incompatibility issue with Windows 7. On the other hand, all versions of 32-bit Windows come with a built-in 16-bit emulator. So the users using 32-bit Windows systems will have no problem running ODE Architect on them.

Also, note that Windows 7's Windows XP mode does not work for ODE Architect. The Windows XP mode is a virtual machine environment provided by Microsoft for Windows 7 users as a solution for backward compatibility. In the XP mode, ODE Architect's front-end user interface runs. But whenever it executes the solver, ODE Architect Tool crashes. This problem may be due to the virtual machine's machine code translation method. The users of Windows 7 have no control over the translation method.

2. ODE Architect was written in Delphi programming language.

As we first tried to port the whole ODE Architect package into 64-bit Windows program, we looked into its source code and found that it was written in Delphi. Currently, the Delphi compiler doesn't build 64-bit applications. We could have tried to recompile ODE Architect to 32-bit instead. But this workaround required a CIS person with expertise in Delphi programming and it seemed not feasible to port it within a reasonable time frame as we had neither development documents nor build file (or project file). Besides, even if we were successful in porting ODE Architect into a 32-bit application, it would only solve the incompatibility problem in a limited manner; if the Windows system becomes 128-bit, it will not work again in the future.

\section{Viable Alternatives and the Future}

To provide viable alternatives, considering the issues described in Section 3, we decided to test the ODE Architect tools on different virtual machine environments other than Windows' native virtual machine environment, Windows 7's XP mode. We tried two major virtualization software packages, VMware ${ }^{5}$ by VMware, Inc. and VirtualBox ${ }^{6}$ by Oracle Corporation.

It was confirmed that ODE Architect ran correctly inside 32-bit Windows virtual machines built with both VMware and VirtualBox. Host machines for this test were 64-bit Windows 7 and Mac OS X 10.6.4 which were the latest OS's from both companies at the time of this writing. So if the ODE Architect users use a 64-bit Windows or a Mac, they can obtain VMware player or VirtualBox for free and run Windows XP virtual machine to install ODE Architect inside the virtual machine.

It was a promising result that the 16-bit legacy software could run inside virtual machine environments on 64-bit Windows and Mac host machines. But most 64-bit Windows users would be deterred by the Windows XP installation and its license requirements. So we considered a remote virtual machine alternative to provide pre-configured 32-bit Windows XP environment with ODE Architect already installed on it.

\footnotetext{
${ }^{5}$ http: / / www. vmware.com

${ }^{6}$ http: //www.virtualbox.org/
} 
Based on these facts, we were encouraged to delve into a cloud computing feasibility test for ODE Architect. We tested a free cloud computing service, CloudShare.com, which offers virtual machine environment through the Internet (for more information, see CloudShare Pro service description at http: / / www.cloudshare.com). We confirmed that ODE Architect tools run fine inside the virtual machines on the CloudShare's cloud computing facility. Once a 32-bit Windows virtual machine (i.e. 32-bit Windows XP in our case) is created and ODE Architect is installed in the virtual machine, the invited users can log in to CloudShare.com and run the pre-configured Windows XP virtual machine from inside their browsers using a Java applet or via Remote Desktop Client applications to execute ODE Architect tools.

This approach cleared major hurdles: 64-bit Windows system incompatibility which was the main problem to solve; Windows XP installation and license requirements which were introduced by the virtual machine workaround solution; platform dependency as a bonus because users may now use the ODE Architect tools from Mac and Linux systems.

What remains now is for Wiley, who holds the copyright on ODE Architect, to contact CloudShare or similar commercial cloud computing service to get more information on how to set up the administrative details of such a service and offer it as an option for ODE Architect users experiencing the incompatibility issue.

\section{References}

[1] William Boyce. Elementary differential equations. John Wiley \& Sons, Hoboken N.J., 9th edition, 2009. ISBN 9780470039403.

[2] Courtney S. Coleman Robert L. Borrelli. Differential equations : a modeling perspective. Wiley, New York, 2nd edition, 2004. ISBN 9780471433323. 